\title{
A High Accuracy Method for Measuring Nonlinear Scattering Parameters Qian Zhang ${ }^{1, a}$, Xia Guo ${ }^{2, b}$ and Xueliu Pan $^{3, c}$

\author{
${ }^{1}$ Xi'an Jiao Tong University School of Science, Xi'an 710049, China \\ ${ }^{2}$ Xi'an Jiao Tong University City College, Xi'an 710018,China \\ ${ }^{3}$ Xi'an Jiao Tong University City College, Xi'an 710018,China
}

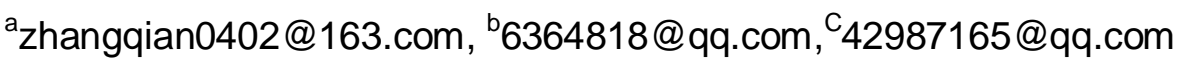

Keywords: Large-signal Scattering functions; Linearization; Artificial neural networks

\begin{abstract}
This paper describes a way of measuring and modeling of microwave transistor nonlinear behavior. We describe a linearization of large-signal scattering functions describing weakly nonlinear device behavior. We illustrate the theory with transistor measurements and design a circuit to test data. Finally, apply Elman Artificial Neural Networks theory to model the large-signal scattering functions of a microwave source in its large-signal operating state.
\end{abstract}

\section{Introduction}

Linear circuit is an idealized concept, but non-linear is its essence. Some circuits such as mixers and frequency multipliers, etc., must be nonlinear. The trend of modern communication applications drive towards high-power level and the development of more complex modulation schemes[1]. This large-signal environment will lead to system components, the device showed strong nonlinear characteristics. At present, nonlinear analysis of high-frequency devices although some progress has been made, such as the a series method, power series expansion method, harmonic balance method, etc., but these are all weak of strong non-linear analysis linear analysis, there is no good way. Therefore, the paper studies a new large-signal analysis, nonlinear large-signal scattering function, can better solve the problem[2].

\section{Measuring Principle}

The Traditional Characterization Methods. The traditional approach to the characterization of nonlinear devices have a lot, but these characterization methods can not be obtained by measuring directly. Here we introduce this new nonlinear scattering function characterization methods can be a good solution to this problem[3].

Linearization of nonlinear scattering function. Single-port linear network as an example to illustrate the scattering function is defined. Activation function of a single-port network with ports to represent the incident wave $a$, and port response function with the port of the reflected wave $b$ to indicate, then the port of reflected waves and the relationship between the port of the incident wave can be expressed using the following formula: $b=F(a), F(a)$ Is a nonlinear function, while it is a complex function, with the first-order approximation to indicate that $b \approx \frac{\partial F(a)}{\partial a} a \circ$ By the complex function theory, we have $b=S a, S=J_{R R}+j J_{I R} \quad$ or $\quad S=J_{I I}-j J_{R I}$

$$
J_{R R}=\frac{\partial \operatorname{Re}(F(a))}{\partial \operatorname{Re}(a)}, \quad J_{I R}=\frac{\partial \operatorname{Im}(F(a))}{\partial \operatorname{Re}(a)}, J_{I I}=\frac{\partial \operatorname{Im}(F(a))}{\partial \operatorname{Im}(a)}, J_{R I}=\frac{\partial \operatorname{Re}(F(a))}{\partial \operatorname{Im}(a)}
$$

The above analysis shows that under certain conditions reflected wave nonlinear one-port network of real and imaginary parts with the incident wave of the real and imaginary parts can be approximated relationship between the linear algebraic equations with a to indicate that this is the one-port linear network linearization. Two-port network shown in Fig. 1. 


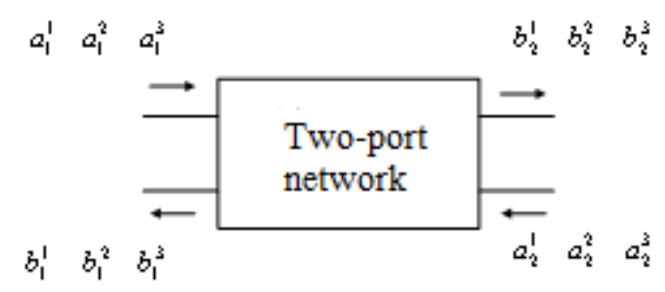

Figure 1. Two-port network

For the two-port network incident wave and reflected waves, respectively, as follows

$$
\begin{aligned}
& {[a]=\left[a_{1}^{1}, a_{1}^{2}, a_{1}^{3}, a_{2}^{1}, a_{2}^{2}, a_{2}^{3}\right]^{T},[b]=\left[b_{1}^{1}, b_{1}^{2}, b_{1}^{3}, b_{2}^{1}, b_{2}^{2}, b_{2}^{3}\right]^{T},} \\
& {[b]=[S \llbracket a]}
\end{aligned}
$$

We can find two-port and dual port incident wave between the reflection wave can also be approximated by a complex algebraic equations to represent. This is the two-port linear network linearization. $\mathrm{S}$ is Nonlinear scattering function matrix[4]. When using normalized incident wave $a_{1}^{m}$ excitation two-port linear network a port, a port in the generated normalized reflected waves $b_{1}^{m}$ and the 2-port transmission wave $b_{2}^{m}$ generated normalized. So the first order approximation of nonlinear network conditions, can be written in the form of:

$$
\left[\begin{array}{l}
b_{1}^{1} \\
b_{1}^{2} \\
b_{1}^{3} \\
b_{2}^{1} \\
b_{2}^{2} \\
b_{2}^{3}
\end{array}\right]=\left[\begin{array}{llllll}
S_{11}^{11} & S_{11}^{12} & S_{11}^{13} & S_{12}^{11} & S_{12}^{12} & S_{12}^{13} \\
S_{11}^{21} & S_{11}^{22} & S_{11}^{23} & S_{12}^{21} & S_{12}^{22} & S_{12}^{23} \\
S_{11}^{31} & S_{11}^{32} & S_{11}^{33} & S_{12}^{31} & S_{12}^{32} & S_{12}^{33} \\
S_{21}^{11} & S_{21}^{12} & S_{12}^{13} & S_{22}^{11} & S_{22}^{12} & S_{22}^{13} \\
S_{21}^{21} & S_{21}^{22} & S_{21}^{23} & S_{22}^{21} & S_{22}^{22} & S_{22}^{23} \\
S_{21}^{31} & S_{21}^{32} & S_{21}^{33} & S_{22}^{31} & S_{22}^{32} & S_{22}^{33}
\end{array}\right]\left[\begin{array}{c}
a_{1}^{1} \\
a_{1}^{2} \\
a_{1}^{3} \\
a_{2}^{1} \\
a_{2}^{2} \\
a_{2}^{3}
\end{array}\right]
$$

$s_{i j}^{m l}$ is the scattering function; $i, j$ are the number of ports; $m, l$ are the number of harmonics.

\section{Experimental data test system}

Structure and composition. Test System Block Diagram Fig.2. below, this test can measure the scattering function thought the first column matrix element.

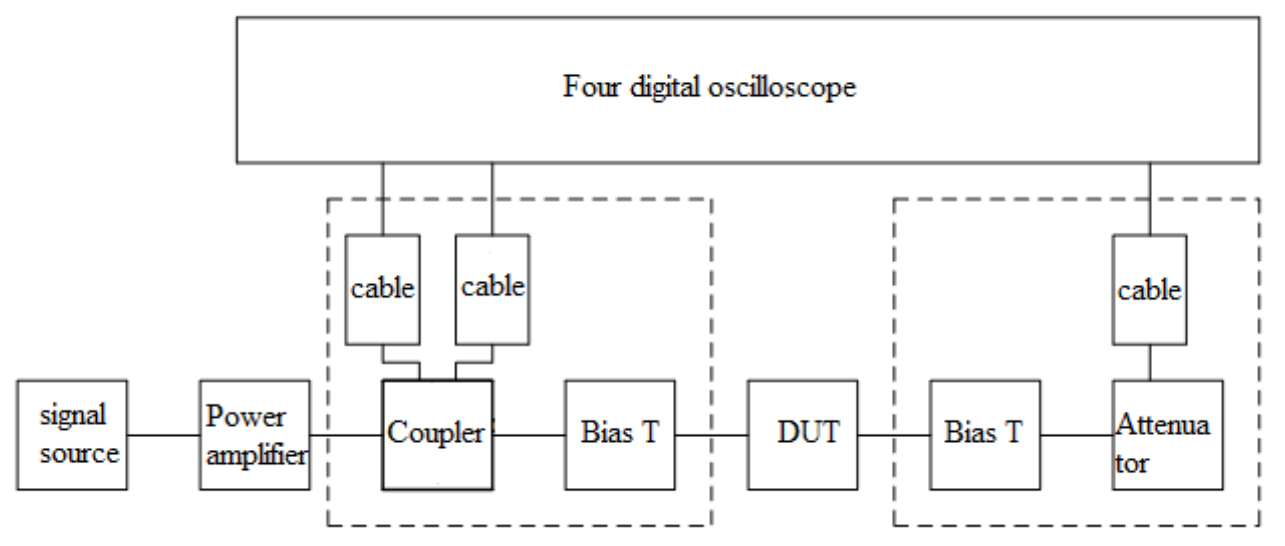

Figure 2. Test System Block Diagram

Test data obtained of $\left|S_{11}^{11}\right|$ as shown in Fig. 3. 


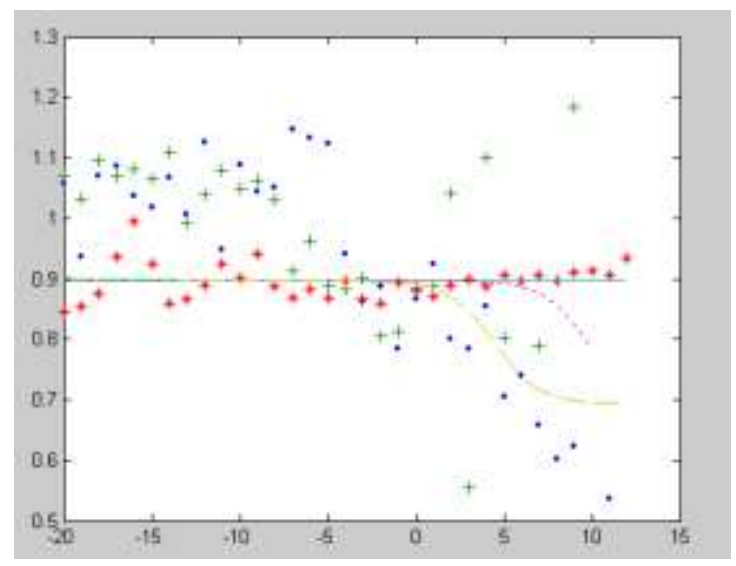

Figure 3. Test data of $\left|S_{11}^{11}\right|$

Horizontal axis represents power, the unit is $\mathrm{dBm}$, vertical axis represents the $\left|S_{11}^{11}\right|$.

Among'.'-100MHz;'+'-150MHz; ‘*'-250MHz; '-’-300MHz

'?-350MHz;'--'-400MHz.

Elman neural network modeling results. Elman Neural network modeling system can be simplified in

Fig. 4.

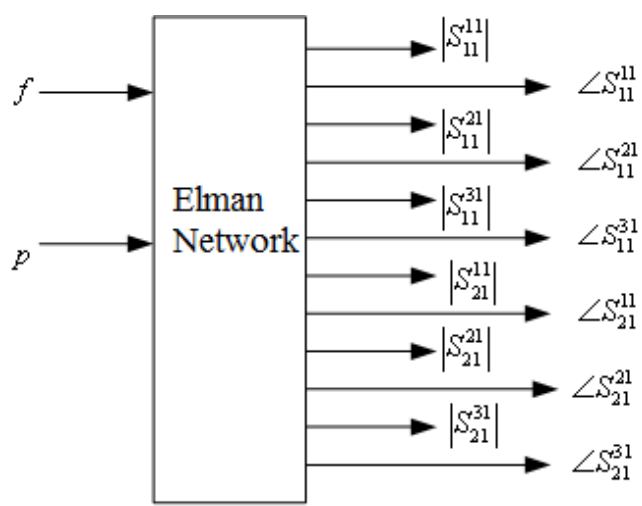

Figure 4. Network input and output variables

12 groups were used as training data amplitude and phase for each model, respectively. Here are some of the modeling $\left|S_{11}^{11}\right|$

to illustrate the problem on. We get a set of data, frequency from

$100 \mathrm{MHz}$ to $400 \mathrm{MHz}, 50 \mathrm{MHz}$ frequency interval of 7 sets of data, power interval change from- $20 \mathrm{dBm}$ to $12 \mathrm{dBm}$, the power interval take $1 \mathrm{dBm}$. Selected six sets of data as training samples to train the Elman network, with $200 \mathrm{MHz}$ of this group test data as validation data to establish whether the Elman network model accuracy. In order to determine the number of first layer neurons, first change the number of neurons from 3 to 20, the simulation analysis of the results shown in Table 6.1.

Table 1 The number of neurons and Error

\begin{tabular}{l|l|l|l|l|l|l|l|l}
\hline Number of neurons & 3 & 4 & 5 & 6 & 8 & 10 & 15 & 20 \\
\hline error & $10 \%$ & $8 \%$ & $5 \%$ & $5 \%$ & $2 \%$ & $5 \%$ & $5 \%$ & $8 \%$ \\
\hline
\end{tabular}

It can be seen that the number of neurons to some extent determines the accuracy of simulation, but does not mean that the more the number of neurons, the better simulation results and accuracy. Here is the modeling results, shown in Fig. 5 and Fig. 6 below. 


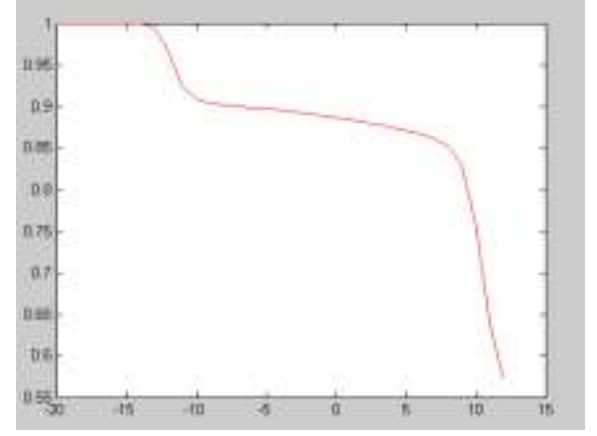

Figure 5. Simulation results

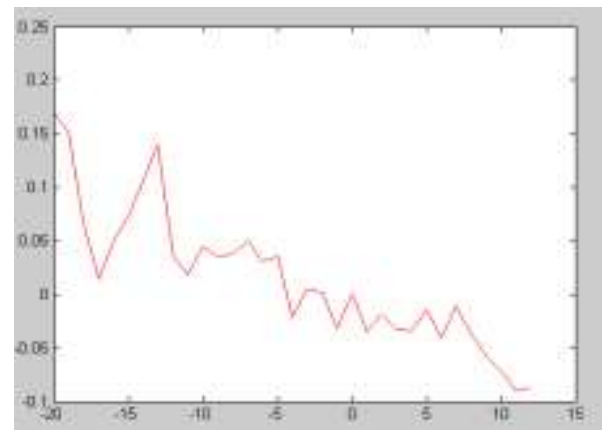

Figure 6. The error curve

Figure 5 is a neural network modeling out of the function graph, the abscissa on behalf of power point, the unit is $\mathrm{dBm}$, vertical axis represents the normalized value.

\section{Conclusion}

If we change the number of neurons found in the number of neurons 8 , the simulation of the curve the best. Neurons more than eight hours, the error did not significantly decline, remains in, so the number of neurons in the best 8 . error of $2 \%$ to control, the use of Elman networks scattering function model can meet the needs of basic accuracy.

\section{References}

[1] W.D. Heiss,R.G. Nazmitdinov: The European physical journal, D. Atomic, molecular, and optical physics, Vol.58(2010) No.1, p.53-56.

[2] Gselmann, E.: Monatshefte fur Mathematik, Vol.169(2013) No.3/4, p.355-370

[3] Hryniv, Rostyslav O.,Manko, Stepan S.:Integral equations and operator theory, Vol.84(2016) No.3, p.323-355.

[4] J. Vivier, A. Mehablia: Chemical and Biochemical Engineering Quarterly, Vol.26(2012) No.3, p.241-248.

[5] Jan Verspecht, Williams D.F, Schreurs,D et al: IEEE Microwave Theory and Techniques, Vol 53(2005),p.1369-1376.

[6] G. Hetsroni, A. Mosyak, E. Pogrebnyak, R. Rozenblit: International Journal of Thermal Sciences, Vol.50(2011) No.6, p.853-868.

[7] Ben-JeLwo, Chia-Liang Teng, Tom Ni,Shirley Lu: IEEE International Electronics Manufacturing Technology Conference(37th ,Johor, 20-22 September 2016), p.1-4.

[8] Alfonso Tierra, Ricardo Romero:Geodezja i Kartografia, Vol.63(2014)No.2,p.199-209.

[9] Martin T. Hagan, Howard B. Demuth, Mark H. Beale: Neural Network Design (Machinery Industry Press , China, 2005).

[10] Nicolae Enaki,Marina Turcan: Advanced topics in optoelectronics, microelectronics, and nanotechnologies V( Constanta, Romania,2010), p. 78211A:1-6. 